\title{
Frühchen mögen Massage
}

\author{
Intensive mütterliche Zuwendung \\ kann die Entwicklung von zu früh \\ Geborenen langfristig günstig beein- \\ flussen.
}

- Brasilianische Forscher haben 73 frühgeborene Babys, mit einem Körpergewicht von 750-1500 $\mathrm{g}$ in zwei Gruppen randomisiert. Die Mütter der Interventionsgruppe wurden angeleitet, viermal täglich für 15 Minuten sanfte Massagen und passive Bewegungen der kindlichen Gliedmaßen durchzuführen. In der Kontrollgruppe wurden keine derartigen Anwendungen eingesetzt.

Die Kinder wurden zwei Jahre lang nachbeobachtet. $\mathrm{Zu}$ diesem Zeitpunkt war das Körpergewicht in beiden Gruppen ähnlich. In der Interventionsgruppe fanden sich jedoch signifikante Vorteile bezüglich der psychomotorischen sowie der mentalen Entwicklung der Kinder. Die Autoren meinen daher, dass diese mütterlichen Massagebehandlungen die Entwicklung Frühgeborener fördert.

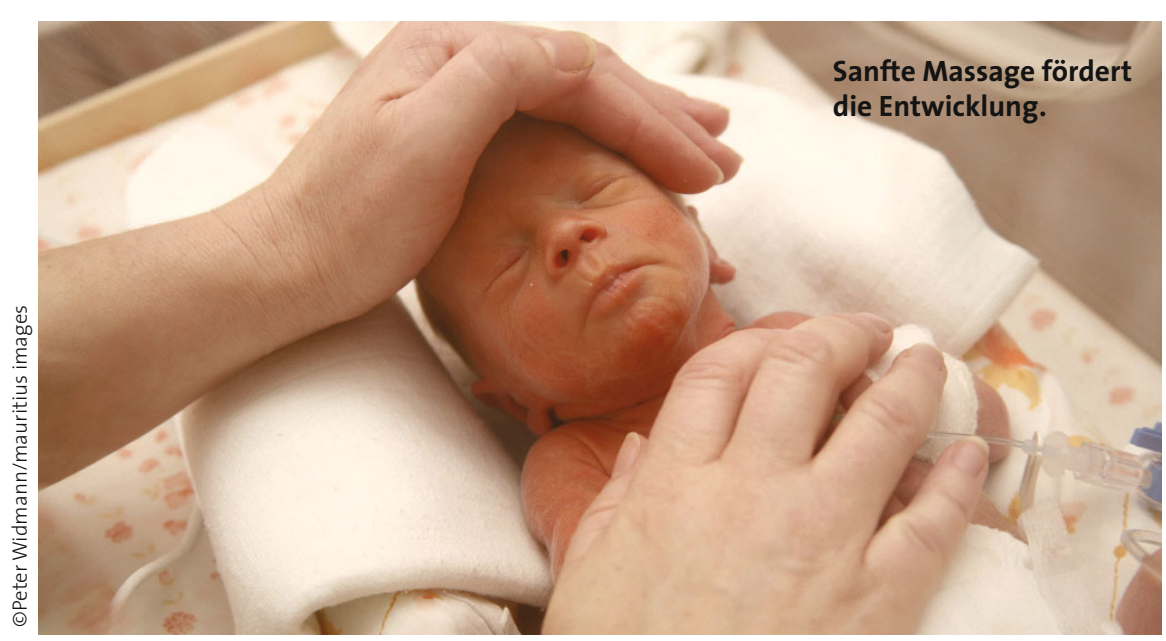

\section{Kommentar}

Mehrere ähnliche Untersuchungen haben bereits recht deutlich gezeigt, dass die mütterliche Massage von untergewichtigen Neu- und Frühgeborenen ihre Entwicklung kurzfristig positiv beeinflusst. Die vorliegende Studie zeigt nun auch, dass solche Effekte langfristig von Bedeutung sind. Die Therapie ist nicht nur effektiv, sondern relativ nebenwirkungsarm und kostengünstig. Ferner mag sie die
Kind-Mutter-Bindung intensivieren und so auch vielen Müttern helfen. Ich sehe daher keinen guten Grund, warum diese Behandlungsform nicht in die allgemeine Routine übernommen werden sollte.

E. ERNST =

- R. S. Procianoy et al.

Massage therapy improves neurodevelopment outcome at two years corrected age for very low birth weight infants. Early Hum. Dev. 2009; doi: 10.1016/j.earlhumdev.2009.12.001

\section{Prügelknabe Hausarzt}

\begin{abstract}
Handgreiflichkeiten gegen Ärzte oder medizinisches Personal sind offensichtlich wesentlich häufiger, als man vermuten möchte. Nur selten haben diese Vorfälle Konsequenzen für die Angreifer, zeigen Zahlen aus Großbritannien. Dort wurde das Thema „Gewalt in Arztpraxen“ kürzlich in einer parlamentarischen Anfrage behandelt.
\end{abstract}

— Ein jüngst publizierter Bericht zeigt, dass im Jahr 2009 britische Hausärzte fast 3500-mal von Patienten angegriffen wurden. Im Vorjahr waren es sogar noch etwas mehr Attacken. Über die Hälfte aller im NHS angestellten Personen geben an, in den letzten fünf Jahren mindestens einmal angegriffen worden zu sein.

Nur in etwa 0,5\% aller Fälle kam es zu einer strafrechtlichen Verfolgung der Schuldigen. Seit November 2009 ist daher ein neues Gesetz in Kraft, das ein straf- rechtliches Vorgehen gegen solche Attacken erleichtert.

\section{Kommentar}

Auch hier ist natürlich Vorbeugen besser als Heilen. Die offiziellen Empfehlungen lauten daher: Potenzielle Angreifer müssen rechtzeitig identifiziert werden (z.B. drogen- oder alkoholsüchtige Patienten). Besondere Vorsicht soll man bei bestimmten Maßnahmen walten lassen (z. B. Verweigerung von Rezepten). Die
Praxisräume sollten so konstruiert sein, dass im Falle eines Falles auch Fluchtwege existieren. Das Personal muss speziell geschult werden, um verbalen oder physischen Aggressionen zu begegnen. Patienten mit einer entsprechenden Vorgeschichte sollten niemals allein untersucht werden. Es scheint fast, als müssten Hausärzte demnächst auch diverse Kampfsportarten beherrschen.

E. ERNST =

\footnotetext{
- L. Anekwe

Figures reveal thousands of assaults on GPs and staff. Pulse 2010; 15 Feb http://www. pulsetoday.co.uk/story. asp? sectioncode $=23 \& \mathrm{~s}$ torycode $=4125082 \& c=2$
} 